\title{
GoPi: Compiling Linear and Static Channels in Go
}

\author{
Marco Giunti $(\bowtie)$ \\ NOVA LINCS, NOVA School of Science and Technology, Lisbon, Portugal \\ marco.giunti@gmail.com
}

\begin{abstract}
We identify two important features to enhance the design of communication protocols specified in the pi-calculus, that are linear and static channels, and present a compiler, named GoPi, that maps high level specifications into executable Go programs. Channels declared as linear are deadlock-free, while the scope of static channels, which are bound by a hide declaration, does not enlarge at runtime; this is enforced statically by means of type inference, while specifications do not include annotations. Well-behaved processes are transformed into Go code that supports non-deterministic synchronizations and race-freedom. We sketch two main examples involving protection against message forwarding, and forward secrecy, and discuss the features of the tool, and the generated code. We argue that GoPi can support academic activities involving process algebras and formal models, which range from the analysis and testing of concurrent processes for research purposes to teaching formal languages and concurrent systems.
\end{abstract}

\section{Introduction}

Concurrent programming is nowadays pervasive to most software development processes. However, it poses hard challenges to the developers, which must envisage and try to solve without automatic support undesired behaviours like security breaches, deadlocks, races, often leading to bugs of substantial impact $[11,22]$. Automated techniques and tools are thus needed to analyse and ensure secure and correct concurrent code. Formal methods have been advocated as an effective tool to analyse and deploy secure communicating programs and protocols [10]. Process calculi, in particular, allow to study prototype analysis techniques that could be embedded into next generation compilers for distributed languages, and to investigate high-level security abstractions that can be effectively deployed into lower-level languages, thus providing for APIs for secure process interaction (e.g., $[2,5])$.

This work is partially supported by the EU Horizon 2020 research and innovation programme under the MSCA RISE grant agreement $N^{\circ} 77823$ (BehAPI), and by Fundacão para a Ciência e a Tecnologia, Ministério da Ciência, Tecnologia e Ensino Superior, via project PTDC/CCI-COM/32166/2017 (DeDuCe). Tool available at: https://github. $\mathrm{com} / \mathrm{marcogiunti} /$ gopi. Demo video available at: https://sites.fct.unl.pt/gopi.

(C) IFIP International Federation for Information Processing 2020

Published by Springer Nature Switzerland AG 2020

S. Bliudze and L. Bocchi (Eds.): COORDINATION 2020, LNCS 12134, pp. 137-152, 2020.

https://doi.org/10.1007/978-3-030-50029-0_9 


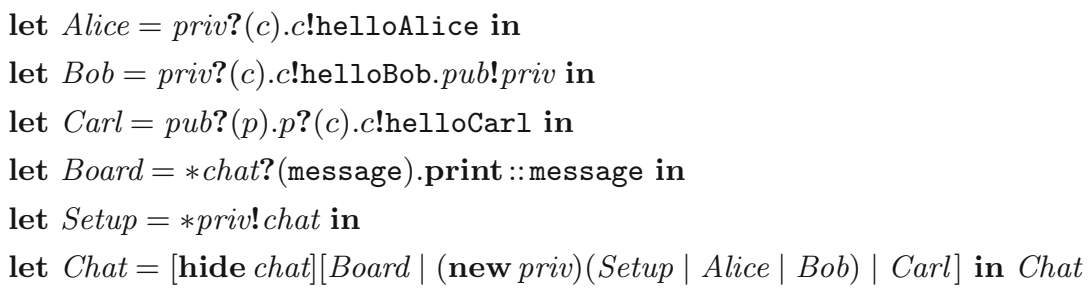

Fig. 1. Suspicious specification of a secret chat in the $L S p i$ language

This paper presents a contribution towards this direction by introducing a fully-automated tool, named GoPi [1], that allows to analyse and run communication protocols specified in a variant of the pi calculus featuring linear channels that must be used exactly once for input and once for output, and static channels that are never extruded. Well-behaved high-level processes are mapped into executable Go programs communicating through message-passing: rather than enforcing the channels' constraints at the target language level, GoPi performs a static analysis of the specification and only generates executable Go code that at runtime preserves the specified invariants. The analysis is based on type inference, while the specification language does not include type decorations. GoPi supports further non-trivial features, which include a contextual analysis of static channels, and deadlock detection on linear channels, at the source language level, and non-deterministic synchronizations, and race-freedom, at the target language level.

The aim is twofold:

- to provide for an automated static analysis of processes described in a variant of the linear pi-calculus without relying on annotations;

- to make available a message-passing runtime system for well-behaved picalculus processes featuring static channels that are never extruded.

\subsection{Message Forwarding Protection}

To illustrate our approach, we consider the case when we want to study the design of a messaging application supporting secret chats ${ }^{1}$ featuring message forwarding protection. To this aim, we analyse an instance of a secret chat that involves three users, and describe the protocol as follows: "Alice, Bob, and Carl share a hidden chat channel with static scope including the users, the board, and a setup process that distributes the channel to the users, where the scope of the channel should never be enlarged". The static scope invariant offers protection against message forwarding, and only processes that are included in the scope of the channel in the specification will be able to ever use the channel at runtime.

\footnotetext{
${ }^{1}$ https://www.viber.com/blog/2017-03-13/share-extra-confidently-secret-chats.
} 
Figure 1 presents a formal specification of the protocol in a variant of the picalculus featuring secret channels. The program is based on message-passing and builds around three main channels: the hidden channel chat, the distribution channel priv, and a public channel pub. Base channels are noted in typewriter. We use !, ?, . , *, and | to indicate output, input, sequence, loop and parallel execution constructors, respectively; channels are created with the new and hide constructors by indicating their scope with parentheses (new) and squares (hide). The print imperative construct allows to print channels. In order to be safe, the program in Fig. 1 should preserve the static scope invariant, that is: the scope of the hidden channel must not be enlarged at runtime. The specification is suspicious since Carl, who is left out of the distribution process, is invited to the chat by receiving the private channel priv from the open channel $p u b$, perhaps because of a bad design choice.

By running GoPi, we verify that, when considered in isolation, the program in Fig. 1 is safe: intuitively, this holds since all processes receiving the hidden channel are included in its static scope (the squares). However, the protocol is flagged as contextually unsafe: the reason is that there exists a process that, once put in parallel with the Chat process, can break the static scope invariant by receiving the hidden channel. That is, because of non-determinism, the private channel priv can be received by a parallel process that is listening on the open channel $p u b$, rather than by Carl, thus allowing a process outside the squares to receive the hidden channel chat. To fix to the program in Fig. 1 we can resort to linear channels that must be used exactly once for input and once for output. By declaring $p u b$ as linear, written as $\langle p u b\rangle$, the protocol SafeChat $\triangleq\langle p u b\rangle$ Chat gains protection from parallel (typed) processes, which are assumed to do not break linearity, and in turn contextual safety, as established by GoPi.

The static analysis is relevant since, in general, detecting if a program may extrude a secret channel by code inspection can be hard, because of channel mobility, and of the arbitrary length of the attack sequence. To see that, take $P \triangleq\left(\right.$ new $\left.a_{1}, \ldots, a_{n}\right)\left([\right.$ hide $\left.c]\left[a_{n} ! c\right]\left|a_{1} ! a_{2}\right| \cdots\left|a_{n-1} ! a_{n}\right| p u b ! a_{1}\right)$, for some $n>1$ : the secret channel $c$ is sent over a restricted channel $a_{n}$, which in turn is sent over a restricted channel $a_{n-1}$, and so on, while the error is that the first channel in the chain, $a_{1}$, is sent over a public channel $p u b$, allowing processes running in parallel with $P$ to receive the hidden channel from $a_{n}$.

\subsection{Related Work}

We briefly discuss work related to the design of the specification language, and to runtime systems for process calculi and Go as a target language.

Language Design. Secret channels have been studied by the author at the language [16] and type [15] level; this work integrates those results by presenting a compiler based on a novel type inference algorithm. The paper [16] presents a variant of the pi-calculus introducing a further operator, hide, that allows to declare channels that can be passed over channels, but cannot be extruded, and studies its behavioural properties. The static scope mechanism is embedded 
in the operational semantics of the language, where a dynamic check ensures that the context cannot receive channels protected by hide. In subsequent work [15], the mechanism is shifted to the level of types by means of a declarative system that enforces the static scope invariant in a standard pi-calculus. These mechanisms, complemented with linear type qualifiers (cf., $[14,18]$ ) and deadlock detection (cf., [17]), are the core of the static analysis performed by the GoPi tool.

Static channels and boundaries in process calculi have been investigated since the origins of this research area [28], and more recently in, e.g., [6,7,26]. The work in [6] has similarities with our approach and introduces a pi-calculus featuring a group creation operator, and a typing system that disallows channels to be sent outside of the group. Programmers must declare which is the group type of the payload: the typing system rules out processes of the form $Q \triangleq($ new $p: U)(P \mid$ $($ new $G)(($ new $x: G[])(p ! x)))$ since the type $U$ of channel $p$ cannot mention the secret type $G$, which is local. In contrast, we do not rely on type decorations and accept process $Q$ whenever $x$ is hidden and $P$ does not allow to extrude $x$, e.g., $P$ does not input on $p$ or distribute $p$. From the point of view of the language design, we share some similarity with the ideas behind the boxed picalculus [26]. A box in [26] acts as wrapper where we can confine untrusted processes; communication among the box and the context is subject to a finegrained control that prevents the untrusted process to interfere with the protocol. Our hide construct is based on the symmetric principle: a process is trusted whenever contexts cannot interfere with the process' protocol, that is contexts cannot enlarge the scope of the hidden channels of the process.

Runtime System. To the best of our knowledge, most interpreters for distributed calculi do not rely on channel-based mechanisms at the target language level; such implementations, pioneered by $[25,27,29]$ for the pi-calculus, are commonly based on simulating non-determinism and concurrency by process interleaving. Previous attempts to develop calculi-inspired languages with native support for channel-over-channel passing include $\mathrm{JoCaml}$ [12], where mobility is now discontinued [23].

Recently, a behavioural static analysis of Go programs based on multiparty session types (MPST, [19]) has been presented in [20,21]. The approach followed in that line of work consists in analysing existing Go programs to ensure stronger properties at compile-time, e.g., deadlock-freedom. None of those works, however, support channel-over-channel passing. Castro et al. [8] introduced a framework to translate distributed MPST written in the Scribble protocol language into a Go API; safety in API's clients is enforced at runtime by generating linearity exceptions. Differently, we obtain safety of Go programs statically by means of type inference of pi-calculus channels.

\section{Structure of the Paper}

Section 2 presents the specification language and the notion of error, and sketches few examples. The next two sections introduce the two main parts of the GoPi 
compiler: the static analyser, presented in Sect.3, and the Go code generator, presented in Sect. 4 . We conclude in Sect. 5 by envisioning possible usage scenarios of GoPi, and by discussing limitations and future work.

\section{The LSpi Specification Language}

This section introduces the syntax of the language processed by the GoPi compiler. We consider communication channels, or variables, $a, \ldots, z$, and processes generated by the grammar:

$$
\begin{aligned}
P, Q::= & x ! v \cdot P|x \boldsymbol{?}(y) \cdot P|(P \mid Q)|\mathbf{0}|[\text { hide } x][P] \mid(\text { new } x)(P)|* P| \\
& \langle a, \ldots, x\rangle P \mid \text { let } X=P \text { in } Q|X| \text { print }:: v
\end{aligned}
$$

Most operators are standard for message passing languages, with some exceptions. We have primitives for sending and receiving channels and continuing as $P$, noted as $x ! v \cdot P$ and $x ?(y) . P$, respectively, for parallel composition, noted $P \mid Q$, for inert processes, noted $\mathbf{0}$, for channel creation, noted (new $x)(P)$, for process variables, noted $X$, and for assigning processes to process variables, noted let $X=P$ in $Q$. The hide operator is the main feature of the language and shall be interpreted as follows: [hide $c][P]$ declares that the fresh channel $c$ should be confined into the (fixed) square brackets even when process $P$ interacts with other processes. In the pi-calculus jargon, this is better summarized by the sentence: "scope extrusion of channel $c$ is disallowed". The other crucial feature is the linear channel declaration $\langle a, \ldots, x\rangle P$, which declares that each of the channels $a, \ldots, x$ must be used exactly once for input and once for output. Loops are programmed with the construct $* P$, which executes $P$ forever. The construct print:: $v$ supplies an imperative command to observe the channel $v$.

We assume the usual notions of free and bound variables and process variables, which we deem pairwise distinct by following the Barendregt convention, and let $x$ be bound in [hide $x][P]$, (new $x)(P)$, and $a ?(x) . P$, and be free otherwise, and $X$ be bound in let $X=P$ in $Q$, and free otherwise. The process let $X=P$ in $Q$ is acyclic whenever $X$ is not free in $P$, and $P, Q$ are acyclic; the remaining cases are homomorphic. We only consider acyclic processes not containing free process variables. We will often avoid training nils, use the - variable wildcard, and refer to channels not used in input or output as to base values, and write them in typewriter style, when convenient.

\subsection{Runtime and Errors}

GoPi allows to run LSpi processes by mapping well-behaved processes into executable Go programs. At a more abstract level, the semantics of the language is provided by translating LSpi processes into standard (typed) picalculus processes: intuitively, the hide construct is mapped into a restriction and has standard semantics (cf., [15]), while linear annotations are separated from processes and used in the static analysis. For instance, the specification 
[hide $c][a ! c] \mid a ?(x) . P$ declares that $c$ should be confined in the squares, while at runtime $P$ can receive the restricted channel $c$ : therefore this process is unsound and should be rejected at compile-time.

LSpi programs can contain three kind of errors, all detected by the GoPi compiler:

(A) channels declared as hidden that can be received by processes outside the static scope of the channels;

(B) channels declared as linear that are not used exactly once for input and once for output;

(C) channels declared as linear that at runtime give rise to deadlocks.

Examples. Process Chat in Fig. 1 does not contain errors. In contrast, process Chat $\mid P$, where $P \triangleq p u b ?\left(x_{\text {priv }}\right) \cdot x_{\text {priv }} ?\left(x_{\text {chat }}\right) \cdot Q$, is an error of kind A: there is a sequence of reductions which leads to the instantiation of the variable $x_{\text {chat }}$ in $Q$ with the hidden channel chat, that is the channel chat can be received by a process outside its static scope. Because of that, GoPi flags Chat as contextually unsafe. Process SafeChat $\triangleq\langle p u b\rangle$ Chat does not contain errors, and is contextually safe, as we will see in Sect. 3: intuitively, this holds since process $P$ above is no longer a valid (typed) opponent, because channel pub is linear and cannot be accessed by the context.

To see an example of an error of kind B, take process $\langle$ priv $\rangle$ Chat, where channel priv is declared as linear. The linear invariant does not hold, because channel priv is used three times in input, by Alice, Bob and Carl (through delegation), respectively, and an unbound number of times in output, by process Setup.

Typical errors of kind $\mathrm{C}$ are processes containing self-deadlocks, which arise when a linear input (output) prefixes a continuation containing the matching output (input), and processes containing mutual deadlocks. The variant of process Chat below, where an ack is sent after sending channel priv over channel $p u b$, and where channels $a c k$ and $p u b$ are linear, contains a mutual deadlock:

$$
\begin{aligned}
& \cdots \quad \text { let } B o b=\text { priv? }(c) . c \text { !helloBob.pub!priv.ack!ok in } \\
& \text { let } C a r l=a c k ?(x) . c o n f i r m ! x \cdot p u b ?(p) \cdot p ?(c) . c ! \text { helloCarl in } \cdots \text { in } \\
& \text { let } C h a t A c k=\langle a c k, p u b\rangle \text { Chat in ChatAck }
\end{aligned}
$$

At runtime the continuation of process $B o b$ will be stuck on the output on the linear channel $p u b$, which can be only unblocked by Carl, because $p u b$ is linear and must be used exactly once for input and once for input. Since Carl, in turn, is blocked on the linear channel $a c k$, the process will deadlock.

An interesting example of security error is process FSA below, which abstracts a forward secrecy attack. Process FSA distributes a secret channel $c$ on a private channel $a$, sends a password on $c$, and afterwards releases channel $c$ on a public channel $p u b$ :

$$
F S A \triangleq(\text { new } a)\left([\text { hide } c]\left[a ! c . c ! \text { pwd } \mid a ?(x) . x ?\left({ }_{-}\right) \cdot p u b ! x\right]\right) \mid p u b ?(z) \cdot Q
$$




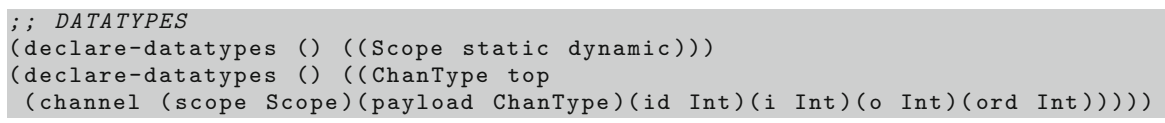

Fig. 2. LSpi types in the SMT-LIB language

By considering that a hide is mapped into a new at runtime, process FSA might be interpreted as secure, because the context cannot observe the exchange over the restricted channel $c$, and in turn cannot retrieve the password. However, preserving the invisibility of restricted communications when pi-calculus processes are deployed in open, untrusted networks is problematic, exactly because of scope extrusion (cf., [3]), and eventually leads to complex solutions based on cryptographic protocols relying on trusted authorities (cf., [5]). For these reasons, we advocate that processes relying on dynamic scope restriction for security should be rejected (cf., $[15,16]$ ). In fact, process FSA contains an error of kind A, because at runtime the secret channel $c$ can be received by a process outside the squares, that is $c$ can be received from $p u b$.

The forward secrecy attack hints on how to use secret channels to develop more secure programs: whenever a secret is sent over an hidden channel of an error-free process, the secret will be unknown outside the static scope of the hide declaration. Process FSecret is one of such secure programs, where we note that the distribution channel $a$ can occur in processes outside the scope of the hide:

$$
\text { FSecret } \triangleq(\text { new } a)(\text { new } b)\left(\left([\text { hide } c]\left[a \text { !c.c!pwd } \mid a ?(x) . x ?\left(_{-}\right)\right]|b ! a| b ?\left({ }_{-}\right)\right)\right)
$$

\section{Static Analyser}

The static analyser is based on the type inference of LSpi channels and is implemented as an automatically generated constraint system written in the $S M T-L I B$ language [4], and decided through the $Z 3$ theorem prover [24]. Notably, the constraint system does not make use of quantifiers.

Figure 2 presents the syntax of the type of LSpi channels, named ChanType: base values are represented by the top constructor, while channels are built with the channel constructor receiving six arguments, where the last three (integer) constructors are for linearity. Type inference of a process $P$ relies on a set of allowed identifiers (cf., $i d$ ), which are the type identifiers that each input process is allowed to receive. Roughly, the static scope analysis is based on this technique.

To illustrate, consider the encoding ${ }^{2}$ of the forward secrecy attack FSA in (2); the input on $a$ is allowed to receive both (dynamic) channels tagged with 0 and the static channel identified by $i d_{c}$, while the input on $p u b$ can only receive channels tagged with 0 :

$$
\text { (new } \left.\left.\left.a: \text { dyn @0)((new } c: \text { stat@ } i d_{c}\right) a ! c . c ! \text { pwd } \mid a ?(x) . x ?\left(_{-}\right) . p u b ! x\right)\right) \mid p u b ?(z) . Q
$$

\footnotetext{
2 The main rationale is that a new is mapped into a new with a dynamic type tagged with 0 , while a hide is mapped into a new with a static type tagged with a positive identifier.
} 
The corresponding SMT-LIB assertions generated by GoPi enforce the invariants for $a$ and $p u b$ through their payload, where the randomly generated identifier that instantiates $i d_{c}$ is 345 :

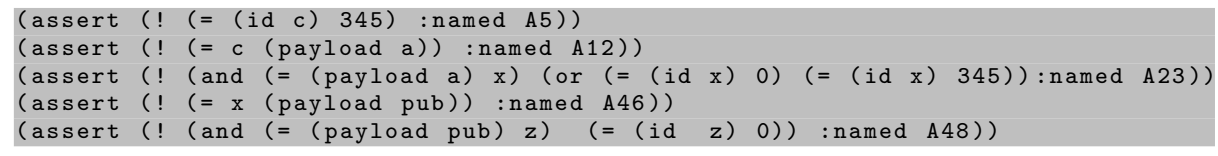

These assertions make the model UNSAT, as expected, because by transitivity we obtain $345=0$ : that is, the variable $z$ bound by the input prefix on channel $p u b$ should have $i d$ equal to 0 , while it has the $i d$ of the static (hidden) channel.

\subsection{Contextual Safety}

Contextual safety is analysed by resorting to auto-generated catalysers (cf., [9]) of order $n$, that are processes that can both inject and receive channels, on which they inject and receive channels, and so on, with depth $n$. Catalysers are put in parallel with the process in order to collect the process' global constraints, as if the process was immersed in an arbitrary (typed) context. The contexts under consideration are those that respect the linearity invariants of the process: that is, we generate catalysers from the unrestricted free variables of the process.

To see an example of catalyser, consider process Chat in Fig. 1, where we note that the only unrestricted free variable of Chat is $p u b$. The catalyser below is generated by following the structure of Chat and by matching each input (output) on $p u b$ with an output (input) on $p u b$ with depth three, which is the maximum order of Chat, where $f$ is a randomly generated channel distinct from any channel in the free and bound variables of Chat:

$$
C a t \triangleq p u b \boldsymbol{?}(x) .(x \boldsymbol{?}(y) . y ?(z) \mid \mathbf{0}) \mid p u b ! f .(f ?(x) . x \boldsymbol{?}(y) \mid f ?(x) .(x \boldsymbol{?}(y) . y \boldsymbol{?}(z) \mid \mathbf{0}))
$$

Process Chat is contextually unsafe because Chat | Cat contains an error: the hidden channel chat at runtime can be received by process $C a t$, which is outside the static scope of the channel (cf., Sect. 2). This is established by GoPi via the generation of the SMT-LIB assertions of Chat $\mid$ Cat, and by discovering that the model is UNSAT; we omit the core assertions, which are similar to those of the forward secrecy attack.

As a further example, consider SafeChat $\triangleq\langle p u b\rangle$ Chat. Given that the set of the unrestricted free variables of SafeChat is empty, we generate an inert catalyser (cf., 0), and in turn obtain that SafeChat is contextually safe because the SMT-LIB model generated from SafeChat $\mid \mathbf{0}$ is $S A T$, that is the parallel composition is error-free.

\subsection{Linearity Analysis}

To enforce linearity, we use the input, output, and order integer constructors, noted $i, o$, and ord, respectively, of the type ChanType in Fig. 2. Input (output) fields contain the number of times that the input (output) capability is used for 
a variable of the given type. Order fields are manipulated by the solver to find an ordering among linear channels.

The linearity analysis is performed by mapping the actual usage of channels into assertions of the constraint system. While analysing processes and generating the corresponding assertions for type reconstruction, we build a usage table that maps channels $x$ to entries of the form $\left(n_{i}, n_{o}, l s\right)$, where $n_{i}, n_{o}$ are integers tracking the usage of $x$ in input and output, respectively, and $l s$ is a list containing the channels where $x$ has been sent. At the end of the process analysis, the contents of the table are transformed into assertions and added to the constraint system.

The SMT-LIB assertions below are an excerpt of the model generated from process ChatAck in (1):

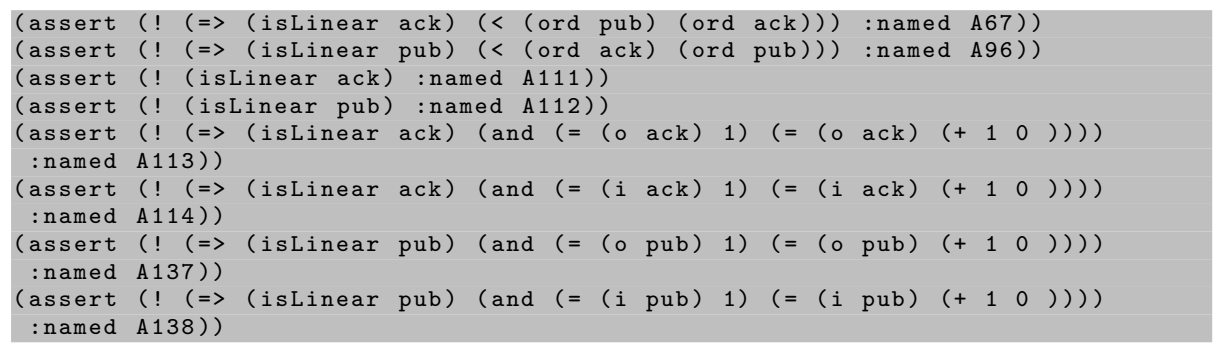

Assertions A111 and A112 come from the linear declaration $\langle a c k, p u b\rangle$ in (1). Assertions A113, A114, A137, and A138 are generated from the usage table, where, for each conjunction, the first entry is the expected value, and the second entry is the actual value. The assertions are satisfiable: that is, each i/o capability of channel $a c k$, and of channel $p u b$, respectively, is used exactly once in (1). The model is UNSAT because the conclusions in the assertions A67 and A96 state that the order of $p u b$ is smaller than the order of $a c k$, and vice-versa. We note that the unsatisfiability of the model prevents the mutual deadlock inside ChatAck (cf., Sect.2).

\section{Go Code Generation}

Given a well-behaved LSpi process, and the type of its channels, GoPi generates executable Go code that is based on the channels' types. Channel types in Go have the following $\operatorname{syntax}^{3}$, where ElementType is any type:

ChannelType = ("chan" | "chan" "<-" | "<-" "chan") ElementType.

We map types in Fig. 2 to types of the form above by ignoring all fields but the payload, and by mapping the top type to string.

The generation of code implementing LSpi processes is not straightforward: while the target language features concurrent goroutines (cf., go $\mathrm{f}(\mathrm{a}, \ldots, \mathrm{z})$ ) that are a natural candidate to represent high-level parallel processes, the whole application's design must be carefully pondered.

\footnotetext{
${ }^{3}$ https://golang.org/ref/spec.
} 


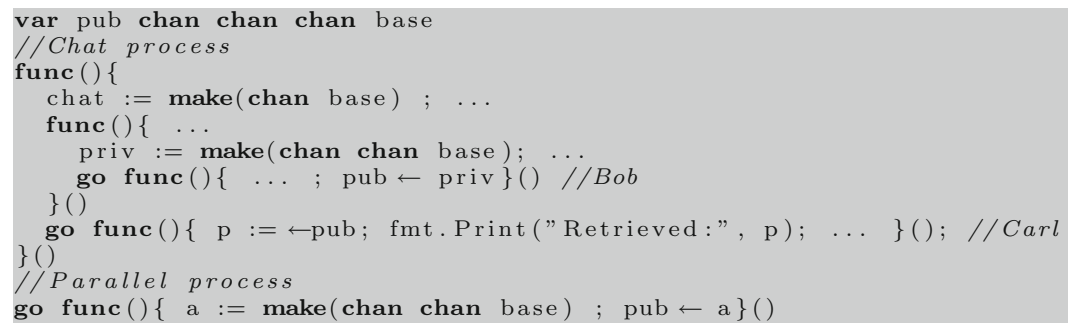

Fig. 3. Naive implementation of the Chat protocol in Go

As a first attempt, we could map input and output constructs of LSpi directly into receive and send primitives of Go, respectively. To illustrate, take the parallel execution of process Chat in Fig. 1 with a process sending a fresh channel $a$ over

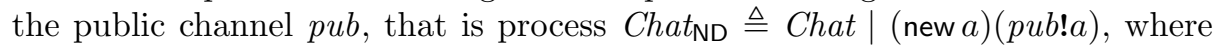
the subscript stands for non-deterministic, since Carl can receive priv from Bob, or $a$ from the parallel process, non-deterministically. Process Chat $t_{\mathrm{ND}}$ would be mapped into Go code of the form outlined in Fig. 3, where we list the parts that are related to the communication over channel $p u b$. The scope of channel chat is grouped by the function call in lines $3-10$, while the scope of channel priv is grouped by the function call in lines $5-8$. The listed processes that are executed concurrently are Bob (line 7), Carl (line 9), and the parallel process (line 12).

While appealingly simple, the implementation in Fig. 3 has at least two main drawbacks:

- in the vast majority of cases, i.e., $\sim 90 \%, p$ is bound to priv, while the probability should be $50 \%$, being receiving priv from pub equally probable to receiving $a$ from $p u b$;

- channels have no name associated, making difficult the interpretation of the output of the program, e.g., "Retrieved: 0xc000022060".

\subsection{Channel Servers}

The envisioned solution consists in using channel servers that take care of input and output requests of clients, while internally managing both non-deterministic synchronizations, and the naming of channels. The access to channel servers is regulated by an API for communication, implemented as methods of a type environment infrastructure; the structure, represented by the typeEnv typed collection in Fig. 4, aggregates channel servers by their order.

Servers are equipped with dynamic arrays, referred as queues, that collect the values concurrently sent on the channel by output clients, and act as a bridge between input and output clients: input clients send requests to the server and receive values sent by output clients and stored in the queue. Non-determinism is simulated through a randomization of queues, and can be pushed forward by 


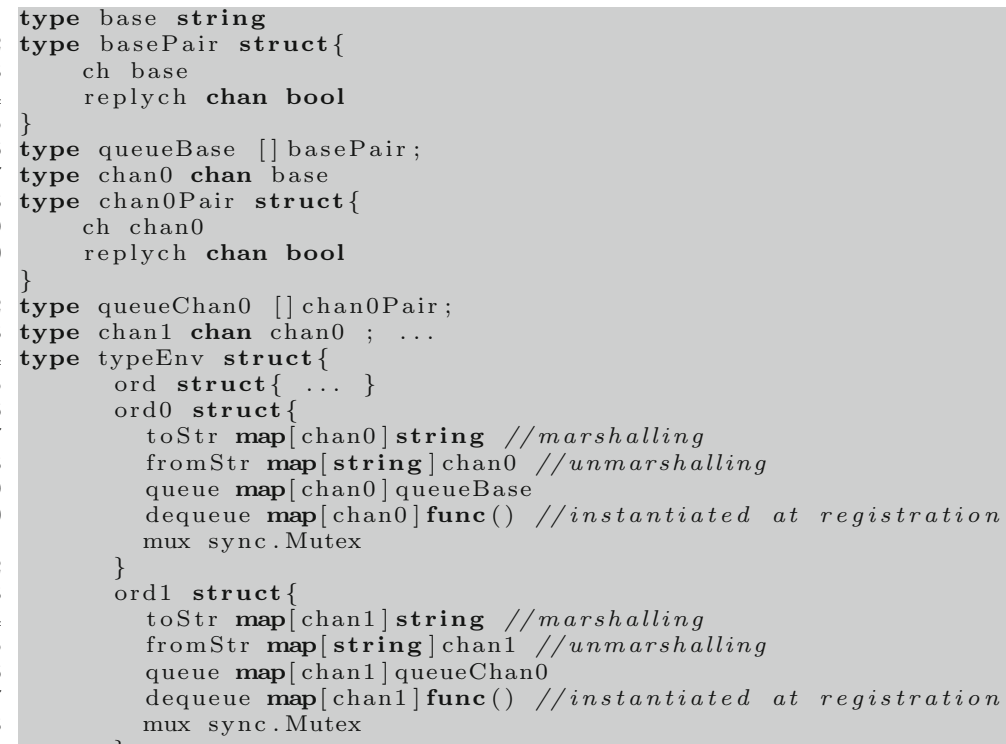

Fig. 4. Type of channel servers

tuning the timeouts in retrieving messages ${ }^{4}$. A mutex regulating the access to queue and dequeue operations prevents data races; this is verified with Go's race detector.

Server Registration. A channel server of order $n \geq 0$ is registered by instantiating the entries of ord $_{n}$ in the (unique) variable $\Gamma$ of type typeEnv (cf., Fig. 4). The procedure to register a channel server of order zero for the name "a", where, by convention, zero is the order of channels conveying base values, consists of five major steps:

1. create a fresh channel $c$ of type $\operatorname{chan} 0$;

2. acquire the lock (cf., line 21)

3. defer the unlock

4. insert the mappings between "a" and $c$ (cf., lines 17, 18)

5 . insert the mapping from $c$ to a function (cf., line 20) that retrieves values from Gamma.ord0.queue[c] (cf., line 19).

\subsection{Clients' Access to Servers}

The channels servers are accessed by clients by means of methods of the variable $\Gamma$ of type typeEnv. The signatures below list the most relevant operations.

4 Non-zero dequeue timeouts are optional, and discouraged for non-academic purposes. 


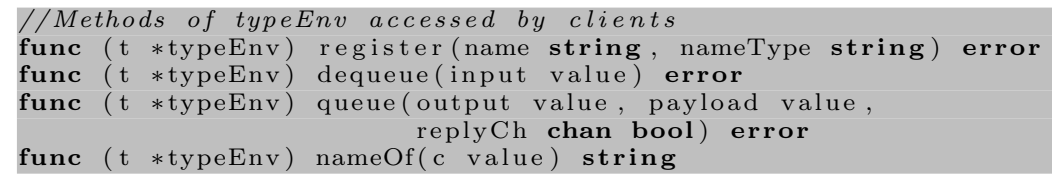

The register method is invoked by clients in correspondence of a new or of a hide declaration, where the second parameter is the order of the declared channel. The dequeue method is called by input clients, where value is an interface implemented by channels and base values. The queue method is invoked by output clients, where the third parameter will be instantiated by a (fresh) ack channel, to enforce synchronous communications. The nameOf method is called by print clients in order to print the string associated to a channel reference.

\subsection{Working Example}

Figure 5 contains the code generated by GoPi for the Chat process (cf., Fig. 1), where we only list the code of clients, being the code of servers invariant. The outer function call generates channel chat and closes its scope. In the body of the call, we have the parallel execution of Board (lines 5-20), of (new priv)(Setup | Alice | Bob) (lines 21-51), and of Carl (lines 52-62). Generation of fresh channels is implemented by a mechanism that uses randomly generated keys, and a counter protected by a mutex, for loops (cf., lines 7, 9, 27, 29).

The code implementing Board invokes the dequeue method of $\Gamma$ (line 10), which triggers the selection of a message $m$ from the queue of channel chat and the dispatch of $m$ over chat. Subsequently, the message is retrieved from chat and printed, where the code in lines 13-17 implements the polymorphic print construct of LSpi. The sending on channel done (line 19) is discussed below.

The code for Setup continuously uses the queue method of $\Gamma$ to send chat over priv (cf., lines 28-33); to enforce synchrony, the write request includes a reply boolean channel that will be unblocked by the server once priv is retrieved in the queue (cf., lines 30, 32).

The code for $B o b$ sends three requests to $\Gamma$ : one dequeue, to retrieve a channel from priv (line 39), one queue, to send the string helloBob over the channel retrieved from priv (line 42), and one queue, to send priv over pub (line 45). Before the exit, a boolean ack is sent over channel done (line 47), to signal that the thread ended. The ack is received by the loop in line 63 , which allows the program to wait for the termination of all threads until a given timeout, to increase the chances to retrieve messages from queues. This mechanism is followed by all threads, regardless of loops.

Finally, the code for Carl sends three requests to $\Gamma$ : one dequeue, to retrieve a channel from pub (line 54), one dequeue, to retrieve a channel $c$ from the channel retrieved from $p u b$ (line 56), and one queue, to send the string helloCarl over $c$ (line 59). 


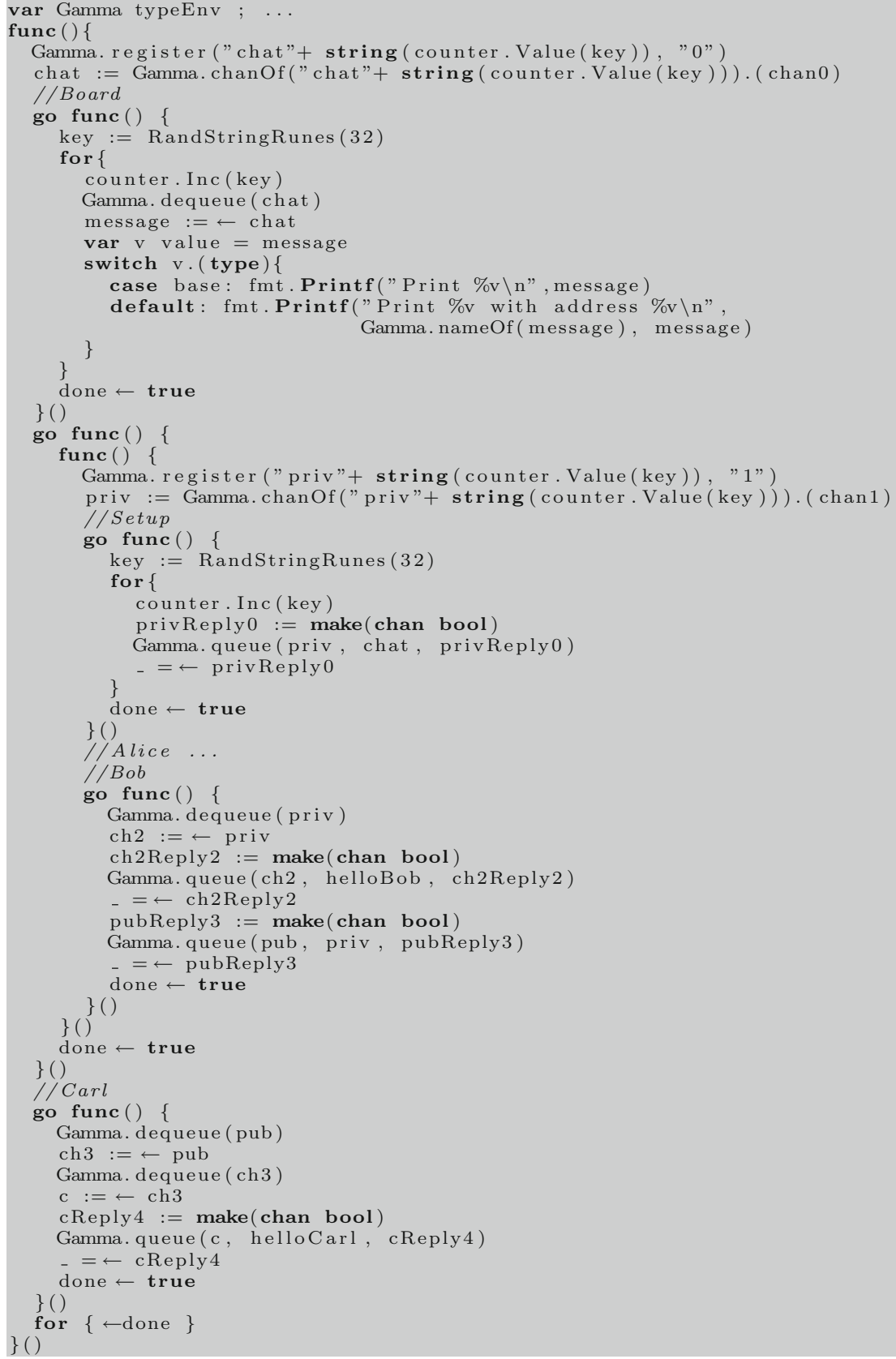

Fig. 5. GoPi's implementation of the Chat process 


\section{Discussion}

GoPi's main aim is to support academic activities involving process algebras and formal models, which range from the analysis and testing of concurrent processes for research purposes to teaching formal languages and concurrent systems.

In this context, we have done some tests ${ }^{5}$ with encouraging results, e.g, GoPi decided the safety of a complex variant of the secret chat protocol of Sect. 1 involving a communication of order seven and more than thirty programming constructs in $0.2 \mathrm{~s}$, producing 600 constraints and a Go file of 1Kloc (cf., [1]). On the Go's side, we ran the code generated from a LSpi process continuously creating, sending and printing fresh channels for one day, without encountering exceptions. With António Ravara, we plan to use GoPi in the course Modelling and Validating Concurrent Systems of the Integrated Master in Computer Engineering, New University of Lisbon, 2020/21.

\section{$5.1 \quad$ Limitations}

The current architecture of GoPi does not allow to separate the static analysis from the generation of the Go code, and in turn to generate code based on type annotations provided by different tools. Another limitation is that modifications of the Go code made by the programmer are lost when the specification is changed, since GoPi does not support annotations of the specification with Go snippets. We also note that the static analysis is not compositional, since to determine whether a process is safe, we perform a contextual analysis.

The information reported in case of failure of the analysis is not parsed into an human-readable format; this limits the usability of the tool.

At the language level, one current limitation is that delegation of partial capabilities of linear channels is rejected, because of issues related to the detection of deadlocks (cf., [17]). Another limitation, which is common in the context of behavioural type systems (cf., [13]), is that deadlocks are detected on linear channels, while unrestricted channels, interpreted as open ports, can give rise to runtime locks caused by decoupled input and output communications.

\subsection{Future Work}

GoPi aims at being an open and live project developing and maintaining a compiler for a language with built-in support for mobility, security, resourceawareness, and deadlock-resolution. In that direction, most limitations outlined above need to be overcome.

The separation of the static analysis and of the generation of Go code, and the readability of the output of the static analysis, appear as the most urgent issues. We believe that both features could be supported in the next release of GoPi, while the presentation of the results of the static analysis could (at least) state a list of channels, and the kind or error encountered (cf., Sect. 2).

\footnotetext{
${ }^{5}$ Testing machine: MacBook 2 GHz i5 8 GB 1867 MHz LPDDR3.
} 
Supporting partial delegation of linear capabilities is another feature that we are keen to support in future releases, while the static analysis may be more involved, because of deadlock detection.

Acknowledgements. The author would like to warmly thank the anonymous reviewers for their competent comments and constructive criticism on a previous draft of the paper, and for providing insightful suggestions in the preparation of this paper.

\section{References}

1. The GoPi Compiler. https://github.com/marcogiunti/gopi. https://sites.fct.unl. pt/gopi

2. 4th Workshop on Principles of Secure Compilation. POPL (2020). https://popl20. sigplan.org/home/prisc- 2020

3. Abadi, M.: Protection in programming-language translations. In: Larsen, K.G., Skyum, S., Winskel, G. (eds.) ICALP 1998. LNCS, vol. 1443, pp. 868-883. Springer, Berlin, Heidelberg (1998). https://doi.org/10.1007/BFb0055109

4. Barrett, C., Fontaine, P., Tinelli, C.: The SMT-LIB Standard: Version 2.6. Technical report, Department of Computer Science, The University of Iowa (2017)

5. Bugliesi, M., Giunti, M.: Secure implementations of typed channel abstractions. In: POPL, pp. 251-262. ACM (2007)

6. Cardelli, L., Ghelli, G., Gordon, A.D.: Secrecy and group creation. Inf. Comput. 196(2), 127-155 (2005). https://doi.org/10.1016/j.ic.2004.08.003

7. Castagna, G., Vitek, J., Nardelli, F.Z.: The seal calculus. Inf. Comput. 201(1), 1-54 (2005). https://doi.org/10.1016/j.ic.2004.11.005

8. Castro, D., Hu, R., Jongmans, S., Ng, N., Yoshida, N.: Distributed programming using role-parametric session types in Go: statically-typed endpoint APIs for dynamically-instantiated communication structures. PACMPL 3(POPL), 29:129:30 (2019). https://doi.org/10.1145/3290342

9. Coppo, M., Dezani-Ciancaglini, M., Yoshida, N., Padovani, L.: Global progress for dynamically interleaved multiparty sessions. Math. Struct. Comput. Sci. 26(2), 238-302 (2016)

10. Cortier, V., Kremer, S. (eds.): Formal Models and Techniques for Analyzing Security Protocols, Cryptology and Information Security, vol. 5. IOS Press, Amsterdam (2011)

11. Fonseca, P., Li, C., Singhal, V., Rodrigues, R.: A study of the internal and external effects of concurrency bugs. In: DSN, pp. 221-230. IEEE Computer Society (2010). https://doi.org/10.1109/DSN.2010.5544315

12. Fournet, C., Le Fessant, F., Maranget, L., Schmitt, A.: JoCaml: a language for concurrent distributed and mobile programming. In: Jeuring, J., Jones, S.L.P. (eds.) AFP 2002. LNCS, vol. 2638, pp. 129-158. Springer, Berlin, Heidelberg (2003). https://doi.org/10.1007/978-3-540-44833-4_5

13. Gay, S., Ravara, A. (eds.): Behavioural Types: From Theory to Tools. River Publishers (2017). https://doi.org/0.13052/rp-9788793519817

14. Giunti, M.: Algorithmic type checking for a pi-calculus with name matching and session types. J. Logic Algebraic Program. 82(8), 263-281 (2013). https://doi.org/ 10.1016/j.jlap.2013.05.003

15. Giunti, M.: Static semantics of secret channel abstractions. In: Bernsmed, K., Fischer-Hübner, S. (eds.) NordSec 2014. LNCS, vol. 8788, pp. 165-180. Springer, Cham (2014). https://doi.org/10.1007/978-3-319-11599-3_10 
16. Giunti, M., Palamidessi, C., Valencia, F.D.: Hide and new in the pi-calculus. In: EXPRESS/SOS. EPTCS, vol. 89, pp. 65-79 (2012)

17. Giunti, M., Ravara, A.: Towards static deadlock resolution in the $\pi$-calculus. In: Abadi, M., Lluch Lafuente, A. (eds.) TGC 2013. LNCS, vol. 8358, pp. 136-155. Springer, Cham (2014). https://doi.org/10.1007/978-3-319-05119-2_9

18. Giunti, M., Vasconcelos, V.T.: Linearity, session types and the pi calculus. Math. Struct. Comput. Sci. 26(2), 206-237 (2016). https://doi.org/10.1017/ S0960129514000176

19. Honda, K., Yoshida, N., Carbone, M.: Multiparty asynchronous session types. J. ACM 63(1), 9:1-9:67 (2016)

20. Lange, J., Ng, N., Toninho, B., Yoshida, N.: Fencing off Go: liveness and safety for channel-based programming. In: POPL, pp. 748-761. ACM (2017)

21. Lange, J., Ng, N., Toninho, B., Yoshida, N.: A static verification framework for message passing in Go using behavioural types. In: ICSE, pp. 1137-1148. ACM (2018). https://doi.org/10.1145/3180155.3180157

22. Lu, S., Park, S., Seo, E., Zhou, Y.: Learning from mistakes: a comprehensive study on real world concurrency bug characteristics. In: ASPLOS, pp. 329-339. ACM (2008). https://doi.org/10.1145/1346281.1346323

23. Mandel, L., Maranget, L.: The JoCaml Language, Release 4.01, 14 March 2014. http://jocaml.inria.fr/doc

24. de Moura, L., Bjørner, N.: Z3: an efficient SMT solver. In: Ramakrishnan, C.R., Rehof, J. (eds.) TACAS 2008. LNCS, vol. 4963, pp. 337-340. Springer, Berlin, Heidelberg (2008). https://doi.org/10.1007/978-3-540-78800-3_24

25. Pierce, B.C., Turner, D.N.: Pict: a programming language based on the pi-calculus. In: Proof, Language, and Interaction, Essays in Honour of Robin Milner, pp. 455494. The MIT Press (2000)

26. Sewell, P., Vitek, J.: Secure composition of untrusted code: box pi, wrappers, and causality. J. Comput. Secur. 11(2), 135-188 (2003)

27. Sewell, P., Wojciechowski, P.T., Unyapoth, A.: Nomadic Pict: programming languages, communication infrastructure overlays, and semantics for mobile computation. ACM Trans. Program. Lang. Syst. 32(4), 12:1-12:63 (2010). https://doi. org/10.1145/1734206.1734209

28. Thomsen, B.: Plain CHOCS: a second generation calculus for higher order processes. Acta Inf. 30(1), 1-59 (1993). https://doi.org/10.1007/BF01200262

29. Turner, D.N.: The polymorphic pi-calculus: theory and implementation. Ph.D. thesis, University of Edinburgh (1995) 